\title{
MEG II and Mu3e status and plan
}

\author{
Paolo W. Cattaneo on behalf of the MEG II collaboration ${ }^{1, *}$ \\ André Schöning on behalf of the Mu3e collaboration ${ }^{2}$, \\ ${ }^{1}$ INFN Pavia, Via A. Bassi 6, 27100 Pavia, Italy \\ ${ }^{2}$ Physics Institute, Heidelberg University, Im Neuenheimer Feld 226, 69120 Heidelberg, Germany
}

\begin{abstract}
The MEG experiment searching for lepton flavor violating muon decay, finished data taking successfully in 2013. The final sensitivity of the experiment was $5.3 \times 10^{-13}$, and since the experiment did not find any signal, the upper limit of the branching ratio of the $\mu \rightarrow \mathrm{e}^{+} \gamma$ was set to be $4.2 \times 10^{-13}$ at $90 \%$ C.L.. The MEG II experiment will have a target sensitivity improved by an order of magnitude down to $6 \times 10^{-14}$ with three years data taking. In 2018 after the detector integration, the muon beam data taking took place with a limited number of readout channels. In 2019, the engineering run and the physics run will be started. The Mu3e experiment will search for the lepton flavor violating decay $\mu^{+} \rightarrow \mathrm{e}^{+} \mathrm{e}^{-} \mathrm{e}^{+}$with unprecedented sensitivity. Construction of the detector is going to start in 2020 and for the first phase a single event sensitivity of $2 \times 10^{-15}$ is expected.
\end{abstract}

\section{Introduction}

The standard model (SM) of the elementary particle physics gives a satisfactory explanation of all experimental results in particle physics available today. However, the SM can not explain why there are three generations, the existence of dark matter, many parameters contained in the model itself, so that it is generally believed that the model is a low energy approximation of a more fundamental theory. One approach to investigate possible physics beyond the SM is to use a high intensity beam to detect rare phenomena. One example is to look for charged lepton flavor violation (CLFV) phenomena. CLFV is practically forbidden in the SM. On the other hand, many new physics scenarios like SUSY-GUT or SUSY-seesaw predict large branching ratio of $\mu \rightarrow \mathrm{e}^{+} \gamma$ and/or of $\mu \rightarrow$ eee. Example Feynman diagrams are shown in figure $1 \mathrm{a}$ for new supersymmetric particles and in figure $1 \mathrm{~b}$ for a heavy $\mathrm{Z}$ ' boson in the framework of an extended electroweak sector.

The MEG experiment was designed to investigate the possibility of $\mu \rightarrow \mathrm{e}^{+} \gamma$. Data were taken between 2009 and 2013, and the final sensitivity was $5.3 \times 10^{-13}$. No signal was found and the upper limit of the branching ratio was set to be $4.2 \times 10^{-13}$ at $90 \%$ C.L. [1, 2]. The MEG II experiment is expected to improve the sensitivity by an order of magnitude with three years data taking, down to $6 \times 10^{-14}$ [3].

The Mu3e experiment was proposed in 2013 [7] to search for the $\mu^{+} \rightarrow \mathrm{e}^{+} \mathrm{e}^{-} \mathrm{e}^{+}$decay with an ultimate sensitivity of $1 \times 10^{-16}$. The detector is in its final design phase and construction is expected to start in 2020. Similar to $\mu \rightarrow \mathrm{e} \gamma$, any observation of $\mu^{+} \rightarrow \mathrm{e}^{+} \mathrm{e}^{-} \mathrm{e}^{+}$would

\footnotetext{
*e-mail: paolo.cattaneo@pv.infn.it
} 
Table 1: Resolutions (Gaussian $\sigma$ ) and efficiencies of MEG II compared with those of MEG

\begin{tabular}{lll}
\hline PDF parameters & MEG & MEG II \\
\hline$E_{\mathrm{e}^{+}}(\mathrm{keV})$ & 380 & 130 \\
$\theta_{\mathrm{e}^{+}}(\mathrm{mrad})$ & 9.4 & 5.3 \\
$\phi_{\mathrm{e}^{+}}(\mathrm{mrad})$ & 8.7 & 3.7 \\
$z_{\mathrm{e}^{+}} / y_{\mathrm{e}^{+}}(\mathrm{mm})$ core & $2.4 / 1.2$ & $1.6 / 0.7$ \\
$E_{\gamma}(\%)(w<2 c m) /(w>2 c m)$ & $2.4 / 1.7$ & $1.1 / 1.0$ \\
$u_{\gamma}, v_{\gamma}, w_{\gamma}(\mathrm{mm})$ & $5 / 5 / 6$ & $2.6 / 2.2 / 5$ \\
$t_{\mathrm{e}^{+} \gamma}(\mathrm{ps})$ & 122 & 84 \\
\hline Efficiency $(\%)$ & & \\
Trigger & $\approx 99$ & $\approx 99$ \\
Photon & 63 & 69 \\
$\mathrm{e}^{+}($tracking $\times$matching $)$ & 30 & 70 \\
\hline
\end{tabular}

be a clear sign for physics beyond the Standard Model. But both processes are also highly complementary: the process $\mu \rightarrow$ e $\gamma$ has a higher sensitivity to new physics appearing in loops whereas the process $\mu^{+} \rightarrow \mathrm{e}^{+} \mathrm{e}^{-} \mathrm{e}^{+}$can better probe lepton flavor violating tree level diagrams.

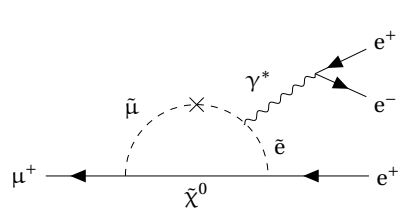

(a) Supersymmetry.

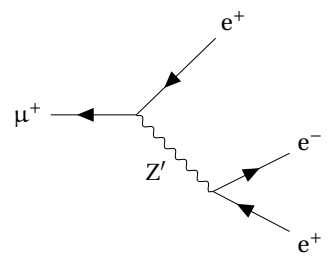

(b) Extended electroweak sector.

Figure 1: The decay $\mu \rightarrow$ eee in extensions of the Standard Model.

\section{MEG II Experiment}

The improvement of the sensitivity in the MEG experiment with additional data taking was already limited by the accidental background. In order to improve the sensitivity further, we needed to upgrade the detector to reduce the dominant accidental background. If the detector performance is sufficiently improved, we can make use of the maximum beam intensity at PSI. To reduce the multiple scattering and the background produced in the target, thinner target will be used in the MEG II. The stopping rate will be $7 \times 10^{7} / \mathrm{s}$, which is more than twice the MEG intensity. The drawing of the MEG II detector is presented in Fig. 2.

The positron spectrometer will use the superconducting magnet again which has a special gradient magnetic field. In order to track $52.8 \mathrm{MeV}$ positron and to reconstruct vertex, angle, and momentum, a new single volume cylindrical drift chamber $(\mathrm{CDCH})$ is built for the MEG II positron tracker which has smaller stereo cells, and thus has more hits resulting in better momentum, angular resolutions. The total length of the drift chamber is $2 \mathrm{~m}$, and the chamber gas will be $\mathrm{He}: \mathrm{iC} 4 \mathrm{H} 10=85: 15$. A new pixelated positron timing counter (pTC) is 


\section{MEG II Experiment}

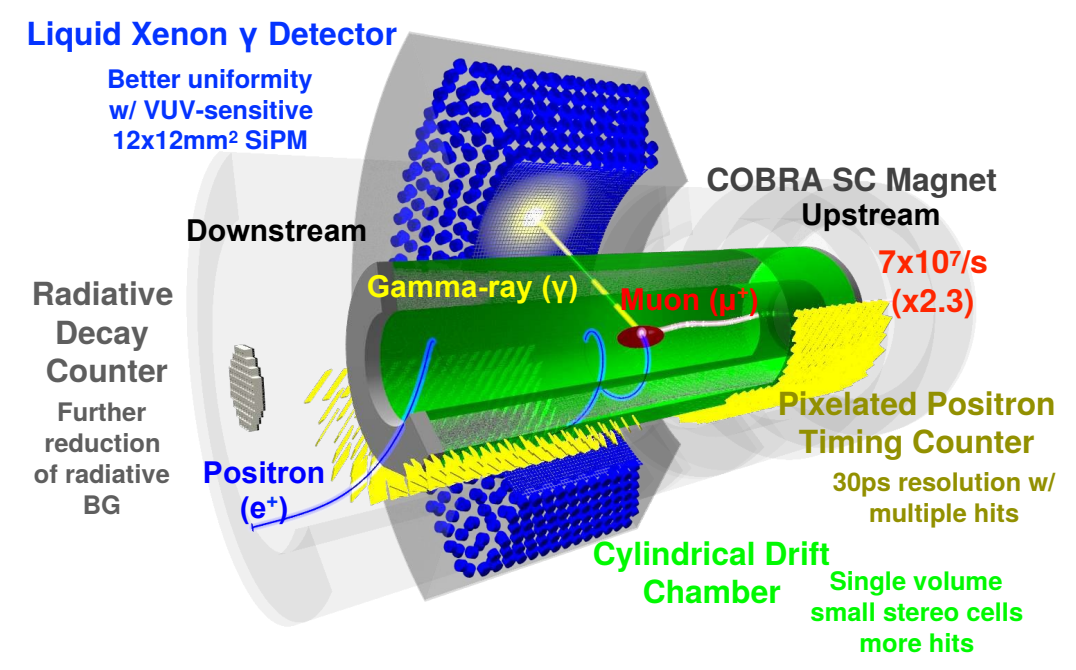

Figure 2: Drawing of the MEG II detector

prepared to detect $52.8 \mathrm{MeV}$ positron time precisely with multiple hits down to $30 \mathrm{ps}$. Segmented 256 scintillator plates with a dimension of $12 \times 4(5) \times 0.5 \mathrm{~cm}^{3}$ readout by SiPMs are installed into both upstream and downstream side. The higher positron detection efficiency is realized by less drift chamber material, high transparency towards timing counter. The inner face of the liquid xenon detector (LXe), which was covered by 2162 inch PMTs, is now replaced with $409212 \times 12 \mathrm{~mm}^{3}$ VUV-sensitive MPPCs newly developed in cooperation with Hamamatsu. This enables better granularity and uniformity of the detected photoelectrons independent of the incident positions, and better energy, position resolutions will be achieved. High energy $\gamma$-rays from radiative muon decays can be the dominant accidental background source because the material inside the drift chamber is reduced, and annihilation in flight can be reduced which is another dominant background. A tagging detector of the RMD to detect low momentum positron with high energy detection in the LXe detector can reduce the RMD background events. The new device for this, called Radiative Decay Counter (RDC), is newly introduced at the downstream side in the MEG II detector. Plastic scintillators readout by SiPM are mainly to detect positron timing, and LYSO crystals with SiPMs are to measure positron energy. Many detectors in the MEG II experiment have finer granularity with SiPMs and more readout channels are required. Experiments like MEG II under high rate environment require waveform digitizers to reduce pileup events. To fulfill these requirements, compact electronics with more functionality including waveform digitizer, simple trigger, bias voltage supply for SiPM, amplifier, called WaveDREAM (Waveform digitizer with DRS4 readout module) are developed at PSI. The online trigger is important to manage high event rate and background suppression, and the FPGA based trigger system is prepared for the MEG II experiment. In Table 1 the resolutions and the efficiencies expected in MEG II are compared with those measured in MEG. Figure 3 shows the MEG II sensitivity as a function of the DAQ time. Conservatively 20 DAQ weeks are assumed for a year. In a 
few DAQ weeks, the current upper limit will be exceeded by the MEG II experiment, and the sensitivity will reach $6 \times 10^{-14}$ in three years.

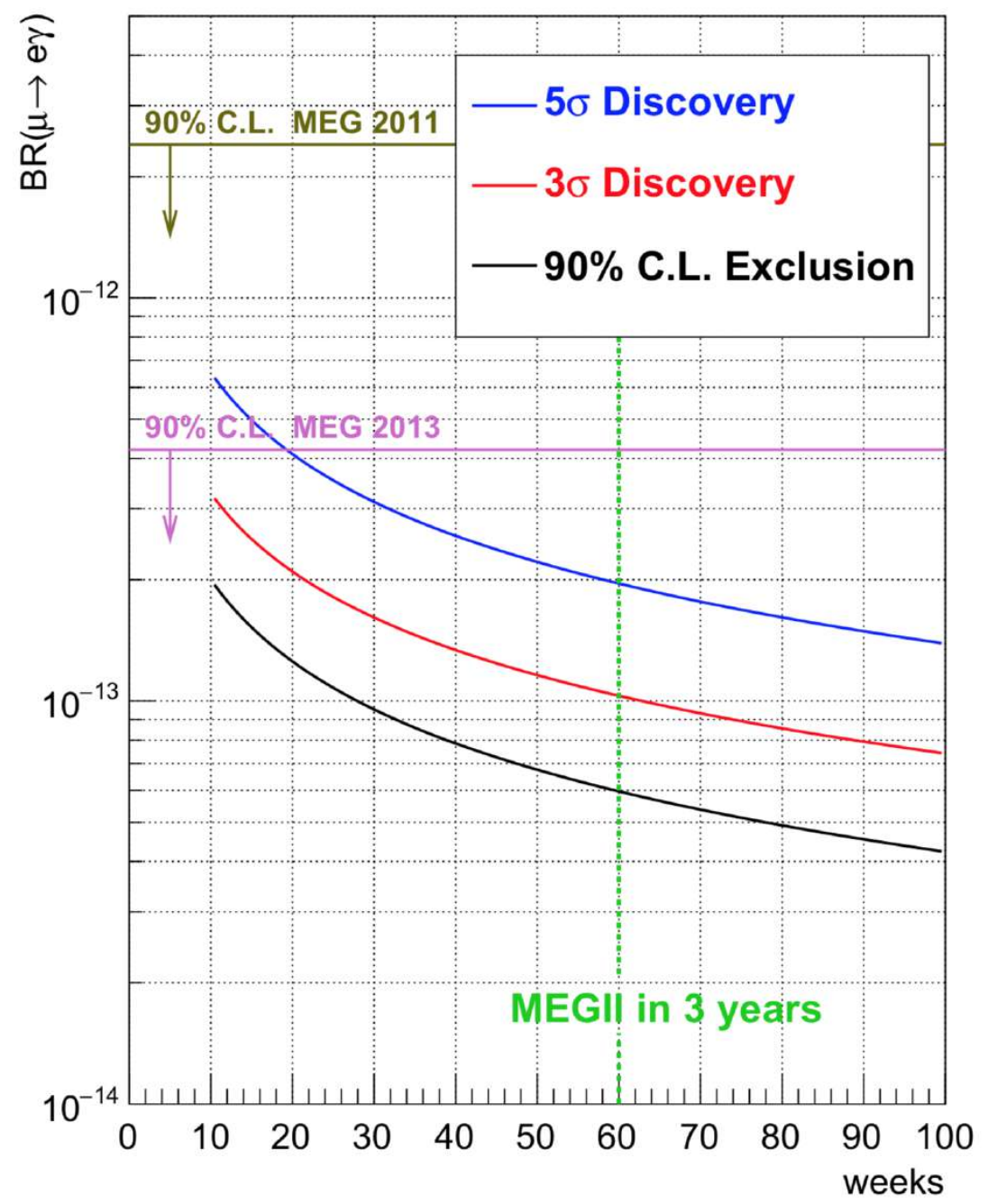

Figure 3: Sensitivity plot as a function of the DAQ time

\section{MEG II Current Status}

The CDCH construction was completed at Pisa in July 2018, and sent to PSI, where it was installed inside the superconducting magnet. The pTC construction has been finished in 2017, and basic performance test was already done with muon beam [5]. The LXe detector construction was also finished in 2017 , followed by detector commissioning. The RDC downstream detector was constructed in 2017 , and the performance test with muon beam was finished in 2017. The electronics development is in the final stage, 96 prototype WaveDREAM boards (1536 channels) were produced, and used to read part of the pTC, LXe, RDC 
detectors in the 2018 pilot run, when all the detectors were integrated in the experimental hall. Based on the this test, the mass production will be started in 2019 [4].

\section{The Mu3e Experiment}

The last search for $\mu^{+} \rightarrow \mathrm{e}^{+} \mathrm{e}^{-} \mathrm{e}^{+}$was performed by the SINDRUM experiment at PSI more than 30 years ago, setting an upper limit on the branching fraction of $1 \times 10^{-12}$ at $90 \%$ confidence limit (C.L.) [6]. The upcoming Mu3e experiment [7] will exploit the same beam line but at higher muon stopping rates of up to $1 \times 10^{8} \mu^{+} / \mathrm{s}$, aiming to increase the sensitivity by more than two orders of magnitude in the first phase. The ultimate goal is to increase the sensitivity by four orders of magnitude by exploiting a new High intensity Muon Beam line (HiMB) providing more than $1 \times 10^{9} \mu^{+} / \mathrm{s}$ in a second phase.

\subsection{Signal and Background}

The signal decay $\mu^{+} \rightarrow \mathrm{e}^{+} \mathrm{e}^{-} \mathrm{e}^{+}$is characterized by the coincidence of two positrons and one electron, originating from the same vertex and the same point in time. Since the muons stop before decay, the energies of the three decay particles equal the muon mass. Two types of backgrounds are important for this search. The radiative muon decay with internal conversion $\mu^{+} \rightarrow \mathrm{e}^{+} \mathrm{e}^{-} \mathrm{e}^{+} \bar{v}_{\mu} v_{e}$ has the identical visible final state as $\mu^{+} \rightarrow \mathrm{e}^{+} \mathrm{e}^{-} \mathrm{e}^{+}$and is irreducible for small neutrino energies. It can only be distinguished from signal by measuring precisely the momentum of the decay electrons ${ }^{1}$ and by rejecting large missing energies carried by the undetected neutrinos. The second type are accidental backgrounds, produced by pile up of two or three muon decays. Bhabha scattering is here a main source as positrons from the Michel decay can kick out electrons from the detector material with large momentum transfer. In combination with other Michel decays the search signature, two positrons and one electron, can be mimicked. This background is efficiently suppressed by means of vertexing and timing, and by applying kinematic constraints. Accurate tracking and very precise timing measurements are therefore crucial for the Mu3e experiment as well as the requirement to sustain high muon decay rates.

\subsection{Experimental Concept}

The Mu3e experiment, see figure 4, is designed to measure low momentum electrons with high precision. Incoming muons are stopped on a hollow double cone target made from Mylar foil. The momenta of the decay products are measured by their bending in the solenoidal magnetic field of 1 Tesla. The tracker design is based on four layers of monolithic active pixel sensors (MAPS). Since the electrons from the muon decay have only low momenta of a few tens of $\mathrm{MeV}$, multiple Coulomb scattering dominates the momentum resolution. Therefore, ultra-light tracking modules with a radiation length of about $0.1 \%$ radiation lengths per pixel layer have been developed. For the Mu3e experiment a dedicated pixel chip (MuPix) has been designed [8-10] which exploits a commercial HV-CMOS process for fast charge collection, providing high rate tolerance and good time resolution below $20 \mathrm{~ns}$. The inner two tracking layers surrounding the target provide precise vertexing. The outer layers allow for the reconstruction of recurling tracks with high momentum resolution. The Mu3e detector is extended by so-called recurl stations upstream and downstream to increase the geometrical acceptance. The minimum transverse momentum acceptance is $10-15 \mathrm{MeV}$.

\footnotetext{
${ }^{1}$ In the following, electrons are used for both, electrons and positrons, if not stated otherwise.
} 


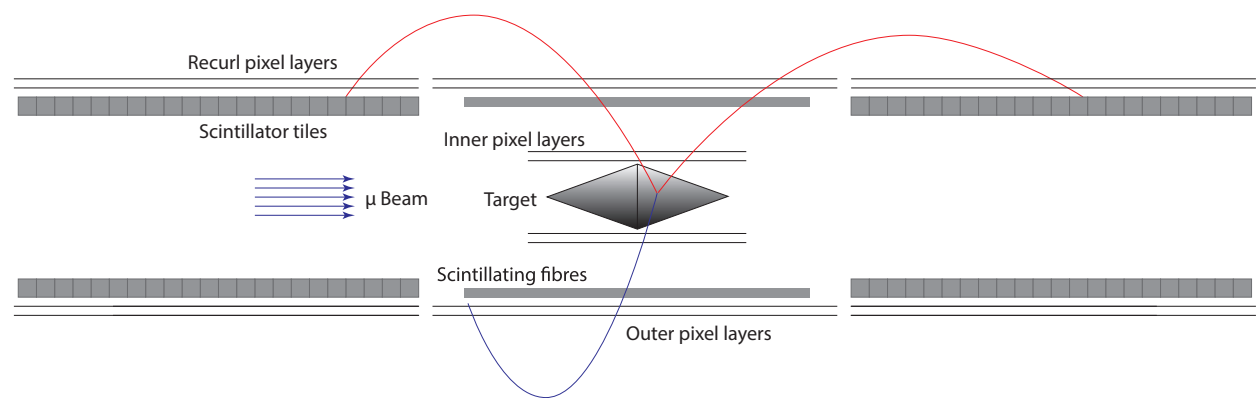

Figure 4: Schematic of the phase I Mu3e experiment in lateral projection with a simulated $\mu \rightarrow$ eee event.

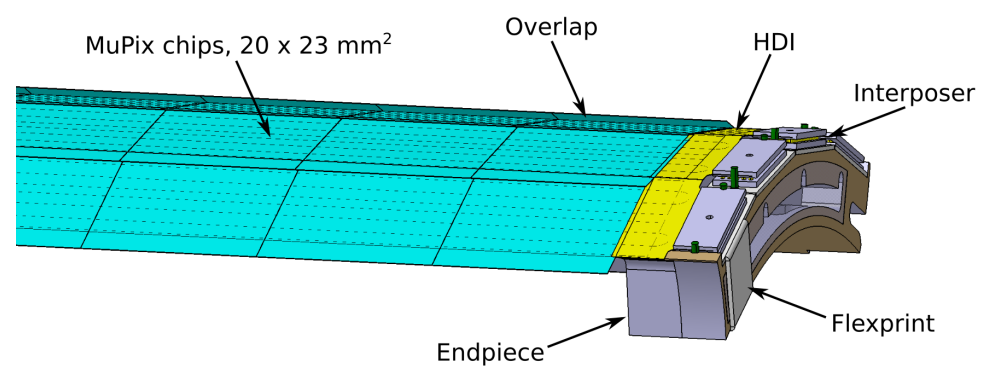

Figure 5: CAD drawing of an outer pixel tracker module: MuPix sensors with a thickness of $50 \mu \mathrm{m}$ are glued and spTAB-bonded on High Density Interconnects (HDI), thus forming ladders. The ladders are electrically readout via interposers and flexprints, and mechanically connected to end-pieces which also provide the helium gas cooling.

A CAD drawing of a Mu3e inner pixel module is shown in figure 5. Several thermomechanical mockups have been successfully built to verify the design.

A three-layer scintillating fiber detector provides sub-nanosecond time resolution for all tracks in the central region of the experiment. Inside the recurl pixel layers, c.t. figure 4, additional timing detectors consisting of scintillating tiles provide a timing resolution better than $100 \mathrm{ps}$. These timing detectors are crucial to reduce accidental background.

The data acquisition is performed without any hardware trigger and all sub-detectors are continuously read out. The data rate, however, is too high for mass storage. Therefore $\mu \rightarrow$ eee candidates are reconstructed online on a filter farm based on commercial Graphical Processing Units (GPUs). The online reconstruction algorithms exploit a fast triplet track fit based on a multiple scattering model [11] and a fast vertex fit to identify the three-prong decay.

\subsection{Sensitivity Studies}

Sensitivity studies have been performed using a detailed GEANT4 simulation of the phase I Mu3e detector, assuming a muon stopping rate of $1 \times 10^{8} \mu^{+} / \mathrm{s}$ and a total run time of 300 days. The $\mu \rightarrow$ eee signal decay is implemented as a three-body decay without any assumptions on the underlying physics. In the event selection, only high quality tracks with at least six hits per track are selected. Constraints are applied on the quality of the vertex fit, 


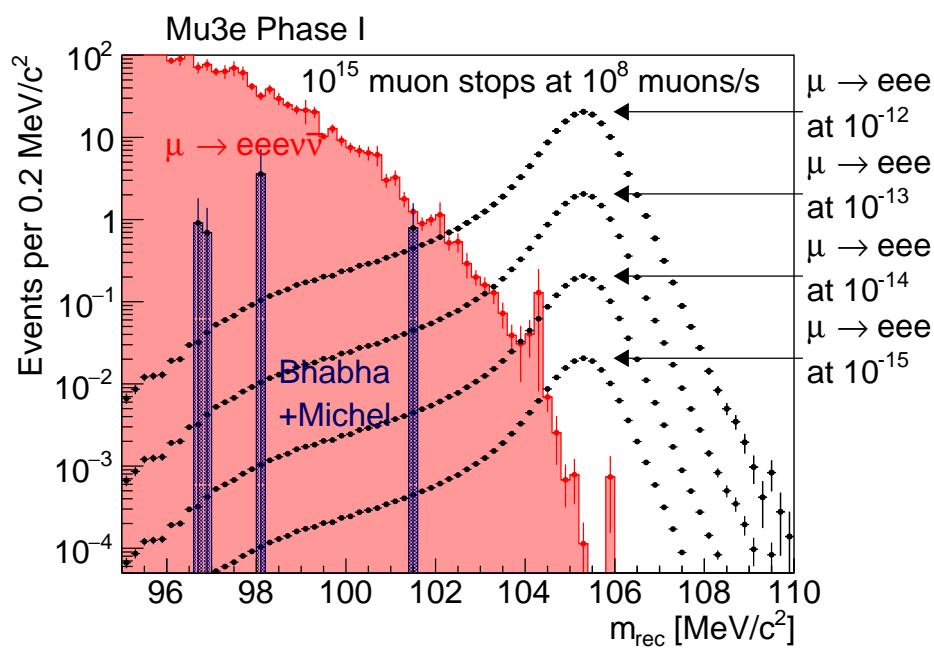

Figure 6: Expected invariant mass distribution of reconstructed $\mu \rightarrow$ eee candidates. Background from the $\mu \rightarrow$ eee $v \nu$ decays is shown in red and the dominant contribution to the accidental background in blue. The signal distribution (black points) is shown for different signal levels.

the distance of the reconstructed vertex to the target, the relative timing between the electron candidates, as well as on the total momentum and invariant mass of the $\mu \rightarrow$ eee candidates. Figure 6 shows the expected invariant mass distribution of the three electrons for the signal and the backgrounds. The signal distribution shows a clear peak at the muon mass, well separated from the background sources. For phase I the Mu3e experiment has an expected sensitivity corresponding to an exclusion limit on the branching fraction of about $5 \times 10^{-15}$ at $90 \%$ C.L..

\section{Mu3e experimental status}

At PSI a new arm of the PiE5 beam line has been installed and commissioned over the last years. This new compact muon beam line will deliver $1 \times 10^{8} \mu^{+} / \mathrm{s}$ to the Mu3e experiment as demonstrated in various measurements. The biggest component of the experiment is the solenoidal magnet providing a homogeneous solenoid field of 1 Tesla. The magnet is produced by company Cryogenic Ltd. and its delivery to PSI is expected for summer 2019. After having proven production readiness, the construction of the main detector components: the pixel tracker, the scintillating fiber detector and the scintillating tile detector will commence in 2020. The current plan foresees installation and commissioning with beams as soon as the detector components become available. In parallel the data acquisition system including the GPU-based filter farm will be installed. First physics results from the search for $\mu \rightarrow$ eee are are expected for 2022 .

\section{Conclusions}

The MEG experiment finished physics data taking in 2013, reaching the most stringent upper limit to date on the $\mu \rightarrow \mathrm{e}^{+} \gamma$ branching ratio, $4.2 \times 10^{-13}$ at $90 \%$ C.L. In order to improve the 
sensitivity by an order of magnitude, the MEG II experiment plans to upgrade all the subdetectors improving their performances. The MEG II detector integration is now in progress. The pilot run in December 2018 fielded all the detectors with a limited number of electronics channels. The mass production of the readout electronics will be finished next year, followed by an engineering run and by a physics run.

The Mu3e experiment will search for the decay $\mu^{+} \rightarrow \mathrm{e}^{+} \mathrm{e}^{-} \mathrm{e}^{+}$at PSI in two phases. By exploiting an existing beam line with a muon rate of $1 \times 10^{8} \mu^{+} / \mathrm{s}$ a single event sensitivity of $2 \times 10^{-15}$ is expected for the first phase. $\mathrm{R} \& \mathrm{D}$ for the Mu3e experiment is going to conclude in 2019 and construction of the detector components will start in 2020, followed by installation and commissioning as soon as detector components are available. First physics runs are expected in 2022. Even higher sensitivities can be reached with an upgraded detector design at the studied High-intensity Muon Beamline which can provide more than $1 \times 10^{9} \mu^{+} / \mathrm{s}$. This would allow to improve the sensitivity by four orders of magnitude with respect to the current experimental limit of $\mathrm{B}\left(\mu^{+} \rightarrow \mathrm{e}^{+} \mathrm{e}^{-} \mathrm{e}^{+}\right)<1 \times 10^{-12}[6]$.

\section{References}

[1] J. Adam et al., [MEG Collaboration], New Constraint on the Existence of the $\mu^{+} \rightarrow \mathrm{e}^{+} \gamma$ Decay, Phys. Rev. Lett. 110 (2013) 201801 arXiv:1303.0754 [hep-ex].

[2] A. M. Baldini et al., Search for the lepton flavour violating decay $\mu^{+} \rightarrow \mathrm{e}^{+} \gamma$ with the full dataset of the MEG experiment, Eur. Phys. J. C76(8), 434 (2016), doi:10.1140/epjc/s10052-016-4271-x, arXiv:1605.05081 [hep-ex].

[3] A. M. Baldini et al., The design of the MEG II experiment, Eur. Phys. J. C78(5), 380 (2018), doi:10.1140/epjc/s10052-018-5845-6, arXiv:1801.04688 [physics.ins-det].

[4] M. Francesconi et al., The WaveDAQ integrated Trigger and Data Acquisition System for the MEG II experiment (2018), arXiv:1806.09218 [physics.ins-det].

[5] M. Nakao et al., Results from Pilot Run for MEG II Positron Timing Counter, Springer Proc. Phys. 213, 237 (2018), doi:10.1007/978-981-13-1316-5 44, arXiv:1808.07279 [physics.ins-det].

[6] U. Bellgardt et al., Search for the Decay $\mu^{+} \rightarrow \mathrm{e}^{+} \mathrm{e}^{-} \mathrm{e}^{+}$, Nucl. Phys. B299, 1 (1988) doi:10.1016/0550-3213(88)90462-2.

[7] A. Blondel et al., Research Proposal for an Experiment to Search for the Decay $\mu \rightarrow$ eee (2013), arXiv:1301.6113 [physics.ins-det].

[8] I. Perić, A novel monolithic pixelated particle detector implemented in high-voltage CMOS technology, Nucl. Instrum. Meth. A582, 876 (2007)

[9] H. Augustin et al., MuPix8 - Large area monolithic HVCMOS pixel detector for the Mu3e experiment, Nucl. Instrum. Meth. A in press, doi:10.1016/j.nima.2018.09.095

[10] H. Augustin et al., The MuPix System-on-Chip for the Mu3e Experiment, Nucl. Instrum. Meth. A845, 194 (2017) doi:10.1016/j.nima.2016.06.095, arXiv:1603.08751 [physics.ins-det].

[11] N. Berger, M. Kiehn, A. Kozlinskiy and A. Schöning, A New Three-Dimensional Track Fit with Multiple Scattering, Nucl. Instrum. Meth. A844, 135 (2017) doi:10.1016/j.nima.2016.11.012, arXiv:1606.04990 [physics.ins-det]. 